\title{
Evaluation of the performance of polished mirror surfaces for the TAMA gravitational wave detector by use of a wave-front tracing simulation
}

\author{
Takayuki Tomaru, Shinji Miyoki, Masatake Ohashi, Kazuaki Kuroda, \\ Takashi Uchiyama, Toshikazu Suzuki, Akira Yamamoto, Takakazu Shintomi, \\ Akitoshi Ueda, Daisuke Tatsumi, Shuichi Sato, Koji Arai, Masaki Ando, Koji Watanabe, \\ Kenji Nakamura, Masahiko Watanabe, Kazuhiko Ito, Izumi Kataoka, \\ Hiroaki Yamamoto, Brett Bochner, and Yaron Hefetz
}

\begin{abstract}
We evaluated the performance of polished mirror surfaces for the TAMA interferometric gravitational wave detector by comparing the experimental results with a wave-front tracing simulation. The TAMA mirror surfaces were polished to a roughness of a few nanometer rms. We confirmed that these polished mirrors do not limit the present TAMA sensitivity and that the target shot-noise sensitivity will be achieved with these mirrors, even if a power-recycling technique is introduced in the next stage of the TAMA. (C) 2002 Optical Society of America

OCIS codes: $\quad 000.2780,000.4430,070.2590,240.5450,240.5770$.
\end{abstract}

\section{Introduction}

The search for gravitational waves (GWs) by use of long-baseline interferometers has started. In the TAMA $^{1,2}$ a Fabry-Perot Michelson interferometric GW detector with a 300-m baseline located in Tokyo,

When this research was performed T. Tomaru, S. Miyoki, M. Ohashi, and K. Kuroda were with the Institute for Cosmic Ray Research, The University of Tokyo, 5-1-5, Kashiwanoha, Kashiwa, Chiba 277-8582, Japan. T. Tomaru (tomaru@post.kek.jp) is now with High Energy Accelerator Research Organization, 1-1, Oho, Tsukuba, Ibaraki 305-0801, Japan as are T. Uchiyama, T. Suzuki, A. Yamamoto, and T. Shintomi. A. Ueda, D. Tatsumi, S. Sato, and K. Arai are with the National Astronomical Observatory, 2-21-1, Osawa, Mitaka, Tokyo 181-8588, Japan. M. Ando is with the University of Tokyo, 7-3-1, Hongo, Bunkyo, Tokyo 113-0033, Japan. K. Watanabe, K. Nakamura, M. Watanabe, K. Ito, and I. Kataoka are with Japan Aviation Electronics Industry Ltd., 3-1-1, Musashino, Akishima, Tokyo 196-8555, Japan. H. Yamamoto is with the Laser Interferometer Gravitational Wave Observatory Laboratory, California Institute of Technology, Pasadena, California 91125. B. Bochner is with the Department of Physics and Astronomy, Hofstra University, Hempstead, New York 11549. Y. Hefetz can be reached at 14 Shoshanim Street, Herzeliya 46492, Israel.

Received 2 February 2002; revised manuscript received 21 June 2002.

0003-6935/02/285913-08\$15.00/0

(C) 2002 Optical Society of America
Japan, several observation runs have already been conducted..$^{3,4}$ The strain sensitivity of the TAMA is $5 \times 10^{-21} \mathrm{~Hz}^{-1 / 2}$ at around $1 \mathrm{kHz}$, which is the highest achieved sensitivity of the present TAMA configuration (as of June 2001). The Laser Interferometer Gravitational Wave Observatory 5 (LIGO) has three sets of optical power-recycling Fabry-Perot Michelson interferometers: Two with baselines of 2 and $4 \mathrm{~km}$ are located in Washington State, and one in Louisiana has a 4-km baseline. All the interferometers can be locked in the power-recycled mode; observations will start soon. Other interferometers, the VIRGO (Variability of Solar Irradiance and Gravity Oscillations; Ref. 6) and the GEO (Geosynchronous Earth Orbit; Ref. 7) will also begin observations soon.

These interferometers require extremely highquality mirrors. If a sufficient quality of mirror polish is not achieved, the designed finesse of various optical resonators will not be attained, and scattered light caused by mirror imperfections (roughness and waviness) will reduce the optical contrast at the output ports. This will decrease the photon shot-noise sensitivity, one of the sensitivity limitations in these interferometers. Optical power amplification techniques, called power recycling, ${ }^{8}$ which are necessary to improve the shot-noise sensitivity, make these requirements even more difficult to achieve because the 
Table 1. Designed and Polished Curvatures of the End Mirrors and the Recycling Mirror of the TAMA

\begin{tabular}{|c|c|c|c|}
\hline Mirror & $\begin{array}{l}\text { Design } \\
(\mathrm{m})\end{array}$ & $\begin{array}{l}\text { Polished Curvature on } x \text { Axis } \\
\text { (m) }\end{array}$ & $\begin{array}{l}\text { Polished Curvature on } y \text { Axis } \\
\text { (m) }\end{array}$ \\
\hline End mirror 1 & 450 & 455 & 456 \\
\hline End mirror 2 & 450 & 454 & 454 \\
\hline Recycling mirror (over whole aperture) & 9000 & 9619 & 9721 \\
\hline Recycling mirror (within beam size ${ }^{a}$ ) & 9000 & 9052 & 9011 \\
\hline
\end{tabular}

${ }^{a}$ The beam radius on the recycling mirror is $8.48 \mathrm{~mm}$.

optical gain of power recycling is sensitive to the optical losses of an interferometer. In the case of the TAMA, the specification for imperfections of the mirrors is several tens of nanometers at low spatial frequencies and subangstrom at high spatial frequencies.

Although all the mirrors in the TAMA were polished and coated and are used in the TAMA (except for a recycling mirror), it has not been conclusively understood whether the performance of these mirrors has limited the sensitivity. In this paper we report on an evaluation of the performance of the TAMA mirrors by comparing the experimental results with a wave-front tracing simulation [a fast Fourier transform (FFT) simulation]. This research was carried out in a collaboration between the TAMA project and the LIGO. Optical phase maps for the imperfections of the TAMA mirrors were measured after polishing by use of a Fizeau interferometer. This report focuses attention on an evaluation of polishing the mirrors without consideration of the coating performance. The FFT simulator used to estimate the mirror performance was originally developed by the VIRGO project ${ }^{9}$ and was later adapted for use in the LIGO. 10,11 The LIGO simulator will be used to model a power-recycling Fabry-Perot Michelson interferometer with an improved calculation speed.

In Section 2 we describe the surface quality of the TAMA mirrors. In Section 3 we describe an evaluation of the polished mirror performance by comparing the experimental results with the FFT simulation. In Section 4 we discuss the mirror performance after a recycling mirror is installed, which is planned for the next stage of the TAMA.

\section{Surface Quality of the TAMA Mirrors}

The polishing of the four main mirrors and the beam splitter was done by Japan Aviation Electronics Industries, Ltd., and the recycling mirror was polished by General Optics Inc. We specified that the polish type (SuperPolish) be able to achieve subangstrom rms microroughness in order to reduce light scatter. Although SuperPolish can achieve small microroughness, ${ }^{12,13}$ it also has a potential problem of increasing the possible distortion of the mirrors.

The substrate of the TAMA mirrors is fused silica, Suprasil P10, produced by Shin-etsu Quartz Products, Co., Ltd. The size of the four main mirrors and the recycling mirror is $100 \mathrm{~mm}$ in diameter and 60 $\mathrm{mm}$ in thickness. The size of the beam splitter is $150 \mathrm{~mm}$ in diameter and $40 \mathrm{~mm}$ in thickness. These diameters were determined so that the diffractive loss of light would be at most 1 part per $10^{6}$ (ppm).

Optical phase maps of the mirror imperfections were measured by use of a Zygo GPI-XP/HR, a Fizeau interferometer that uses 633-nm wavelength light. The apparatus can measure imperfections at only low spatial frequencies. In this study we used phase maps with $256 \times 256$ pixels (spatial-frequency range of between 0.01 and $0.64 \mathrm{~mm}^{-1}$ ) because of the limitation of the computational power.

The near mirrors and the beam splitter are flat, and the end mirrors and recycling mirrors are concave. We first examined the polished curvature of the concave mirrors by fitting the data of the optical phase maps to a spherical surface. Table 1 lists the designed and polished curvatures. The curvature of the recycling mirror within the beam size (the radius is $8.48 \mathrm{~mm}$ ) is almost the same as the design value. Because the requirements for the curvature of the TAMA end mirrors are within a \pm 30 - $\mathrm{m}$ error and the curvature of the recycling mirror must be between 4000 - and 100,000-m curvature, the curvatures of these polished mirrors achieved their requirements.

We next examined small imperfections on the mirror surfaces. Figure 1 shows the imperfections found in the TAMA mirrors. In the concave mirrors the global curvature was subtracted by use of the above fitting data. After subtracting the global curvature from the phase maps, we found that the phase maps for the end mirrors show a Newton's ring pattern arising from interference between the front and rear faces of the mirror. Because this measured pattern causes a large error in the evaluation of the mirror performance, we subtracted it from the phase maps by fitting. In the image shown in Fig. 1, this pattern has been subtracted. The analysis of this pattern is described in Appendix A.

Last, we investigated the polishing level (rms amplitude and uniformity) by using the power spectral density (PSD) of the mirror imperfections. ${ }^{14}$ The calculation method for the PSD of the mirror imperfections ${ }^{15}$ is described in Appendix B. Figure 2 shows the calculated PSDs for the TAMA mirror surfaces derived from their phase maps. Imperfections from high-quality polish obey the equation

$$
P_{\mathrm{av}}(f)=A / f^{q},
$$

where $P_{\text {av }}(f)$ is the PSD, $f$ is the spatial frequency, and $A$ and $q$ are constants. ${ }^{16}$ If the surface imper- 

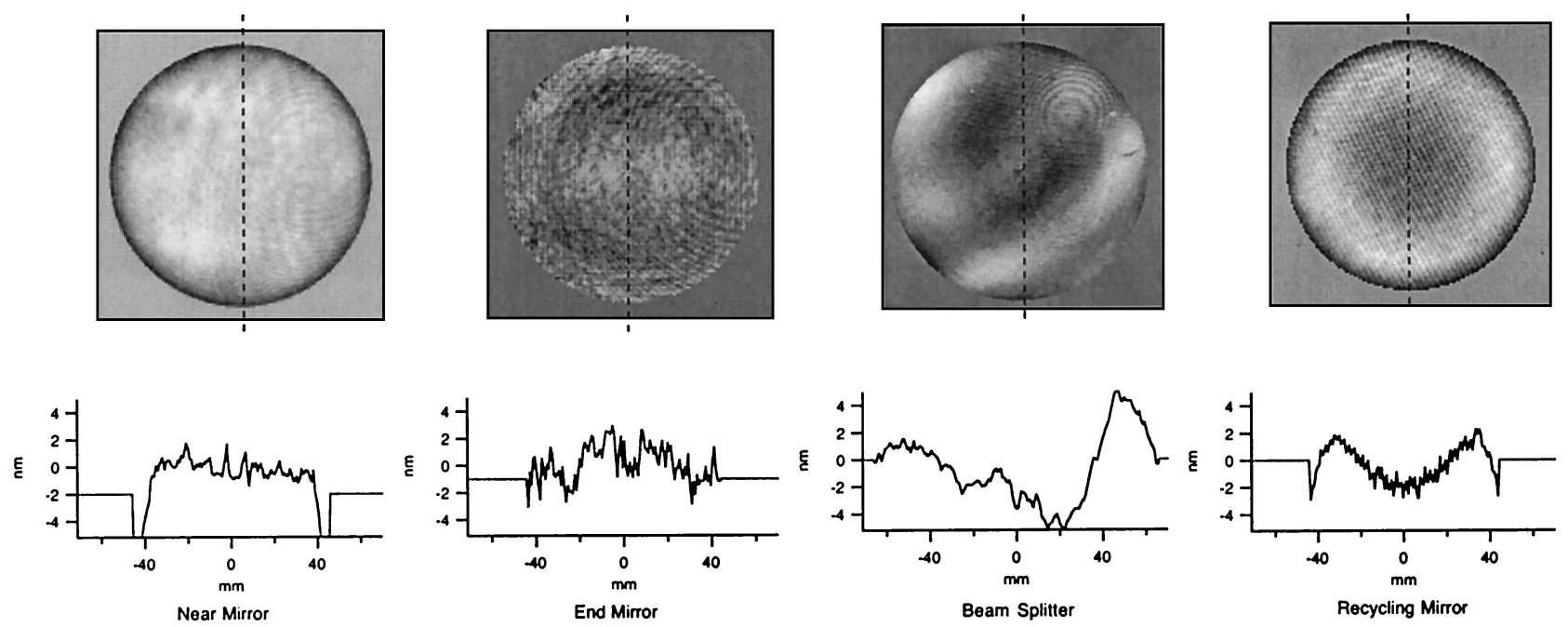

Fig. 1. Imperfection images of the TAMA mirror surfaces. Only one of the two possible images is shown here. The end-mirror image was obtained by subtraction of its global curvature and the Newton's ring pattern. The image of the recycling mirror was obtained by subtraction of its global curvature. The cross-section graphs are images along the dashed lines.

fections have a Gaussian random distribution, the constant $q$ must be between 1 and $3 .^{16}$ We fitted the PSD for the TAMA mirror surfaces to this function. Table 2 lists the $A$ and $q$ parameters, as well as the rms roughness for the TAMA mirror surfaces. These results showed that all the TAMA mirrors had a roughness of a few nanometer rms and had a high uniformity of polish, as evidenced by $1<q<3$.

\section{Comparison of the Experimental Results with the Fast Fourier Transform Simulation}

A useful method to evaluate the performance of mirror surfaces in interferometers is a wave-front tracing simulation. Wave-front propagation is described by the Fresnel-Kirchhoff integral ${ }^{17}$

$$
\begin{aligned}
u(x, y) & =\frac{\mathrm{i} k}{2 \pi L} \iint u_{0}\left(x_{0}, y_{0}\right) \exp (-\mathrm{i} k \rho) \mathrm{d} x_{0} \mathrm{~d} y_{0}, \\
\rho & =\left[\left(x-x_{0}\right)^{2}+\left(y-y_{0}\right)^{2}+L^{2}\right]^{1 / 2}
\end{aligned}
$$

where $k$ is the wave number, $L$ is the separation between the initial and the final planes of light propagation, $\left(x_{0}, y_{0}\right)$ is the position of the wave front on the initial plane, $(x, y)$ is the position of the wave front on the final plane, and $u_{0}\left(x_{0}, y_{0}\right)$ and $u(x, y)$ are the electric light fields on the initial and final planes, respectively. Because this integral is a convolution, the wave-front distortion caused by imperfect mirrors is estimated by a simulation that uses FFTs. The propagated wave front in Fourier space is inversely Fourier transformed just in front of the mirror, and the information concerning the imperfections is impressed on the phase of the light. After the wave front is Fourier transformed again, it propagates to the next mirror. Finally, steady-state electric fields of light are calculated in all parts of the interferometer. ${ }^{10,11}$
Figure 3 shows a model of a GW interferometer of the LIGO's FFT simulator. The carrier beam and the sideband beam must be calculated separately. The length between the mirrors in the simulator is controlled so as to satisfy the resonant or antiresonant conditions for each beam.

Table 3 lists the TAMA parameters. The optical power reflectivity of the near mirror was estimated from the number of coating layers because it was not measured with high accuracy. The reflectivity of the end mirror and the optical loss of the mirror were measured by installation of test pieces of the TAMA mirrors into the $20-\mathrm{m}$ prototype GW interferometer. ${ }^{18}$ The measured optical loss was regarded as the loss caused by imperfections on the mirror surfaces smaller than the pixel size of the phase maps because the beam size of the $20-\mathrm{m}$ interferometer, $4.4 \mathrm{~mm}$ at the waist, is close to the pixel size. Because the present TAMA has no recycling mirror, it was not used in this simulation.

Table 4 shows the results of the experiment and the simulation. The cavity reflectivity is the average between both arms. The cavity reflectivity of the experiment was estimated from a measurement of visibility. In that it is difficult to measure it without the influence of mode mismatching and the sideband, it is assumed that the difference between the experiment and the simulation came from the measurement error. The finesse of the experiment was estimated from the frequency response of the arm cavity. Because the estimated reflectivity of the near mirror can be higher than the real value, the difference in the finesse between the experiment and the simulation can come from the estimation error of the reflectivity. The contrast includes an effect of the sideband. It was measured just after the fourth data-taking run during September 2000. ${ }^{3}$ The simulation result showed that the contribution of the 


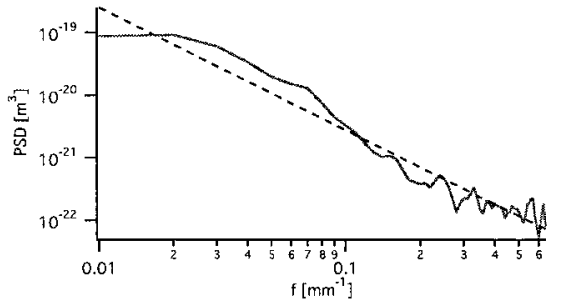

Near Mirror

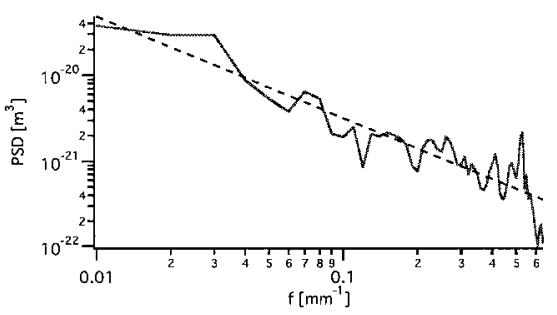

End Mirror

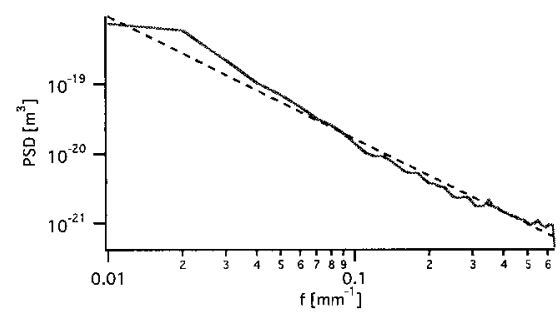

Beam Splitter

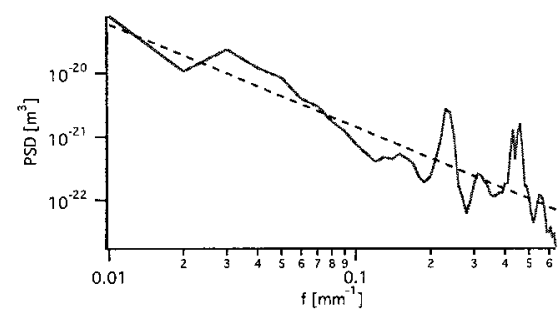

Recycling Mirror

Fig. 2. PSD of the TAMA mirror surfaces. For the near mirror and the end mirror, only one of the two possible images is shown here. For the curved mirrors, the global curvatures were subtracted by fitting. The dashed lines show the function $A / f^{q}$ fitted to the data, where $f$ is the spatial frequency. Parameters $A$ and $q$ are listed in Table 2.

sideband to the contrast is dominant and that of the mirror imperfection is not large.

The most important result is that the estimated sensitivity by the simulation $\left(4.9 \times 10^{-22} \mathrm{~Hz}^{-1 / 2}\right)$ is much smaller than the experimental value $(1.5 \times$

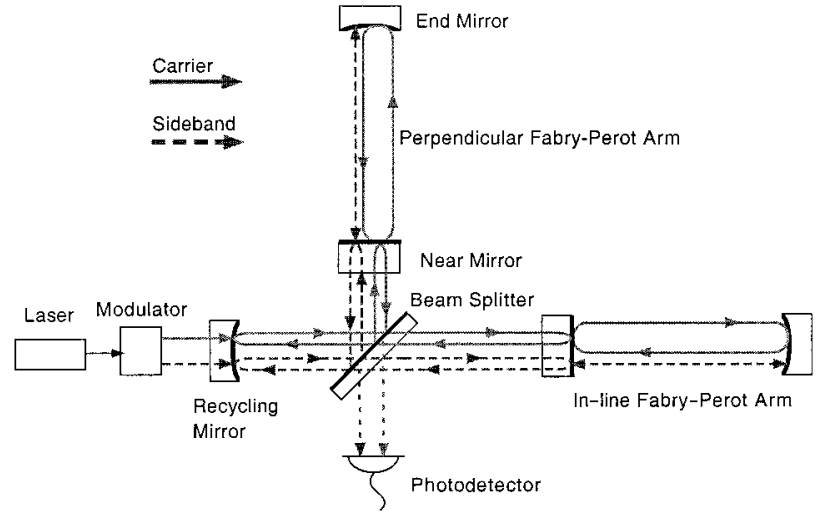

Fig. 3. Model of a GW interferometer used in the FFT simulator. (A power-recycling Fabry-Perot Michelson interferometer.)

$10^{-20} \mathrm{~Hz}^{-1 / 2}$ ), where the sensitivity of the simulation is the photon shot noise at $300 \mathrm{~Hz}$ and that of the experiment and the design include other noises. The experimental sensitivity was taken just after the fourth data-taking run. ${ }^{3}$ This result indicates that the present TAMA sensitivity is not limited by the mirror performance. Also, because the estimated shot noise is much smaller than the design sensitivity $\left(2.0 \times 10^{-21} \mathrm{~Hz}^{-1 / 2}\right)$, we conclude that the surface qualities of the TAMA mirrors are sufficient to achieve the requirements for the first goal of the TAMA.

\section{Studies on the Recycled TAMA}

In this section we discuss the performance of the mirrors for the second configuration of the TAMA, which will have a recycling mirror. The parameters of the calculation are listed in Table 3 . We used the optimized reflectivity of the recycling mirror, in which the reflectivity is equivalent to the cavity reflectivity. The loss of light caused by interferometer control pickoffs was not modeled in this simulation.

Table 5 shows the numerical results. The designed recycling gain is set to a lower value than the value calculated from the design reflectivity of the mirrors. The mirror imperfections cause a reduction in the recycling gain from 19 to 11 for the carrier beam. Because this reduced recycling gain agrees with the value in the case of an ideal recycling mirror, we found that the imperfections of the polished recycling mirror are not important with respect to the performance of the interferometer. The reduction in the recycling gain is caused only by the reduction of the cavity reflectivity. In that the cavity reflectivity for the carrier beam included little higher mode

Table 2. Parameters of the Fit by $A / f^{q}$ to PSD and the rms Roughness for the TAMA Mirror Surfaces ${ }^{a}$

\begin{tabular}{ccccccc}
\hline Parameter & Near Mirror 1 & Near Mirror 2 & End Mirror 1 & End Mirror 2 & Beam Splitter & Recycling Mirror \\
\hline$A$ & $10^{-22.5}$ & $10^{-22.2}$ & $10^{-21.7}$ & $10^{-21.9}$ & $10^{-21.5}$ & $10^{-22.5}$ \\
$q$ & 1.96 & 1.84 & 1.18 & 1.46 & 1.75 & 1.61 \\
rms roughness & $2.0 \mathrm{~nm}$ & $3.7 \mathrm{~nm}$ & $1.5 \mathrm{~nm}$ & $2.0 \mathrm{~nm}$ & $4.6 \mathrm{~nm}$ & $1.4 \mathrm{~nm}$ \\
\hline
\end{tabular}

${ }^{a} f$ is the spatial frequency. 
Table 3. Parameters of TAMA Used in the FFT Simulation

\begin{tabular}{lclc}
\hline \multicolumn{1}{c}{ Parameter } & Quantity & \multicolumn{1}{c}{ Parameter } & Quantity \\
\hline Arm length & $300 \mathrm{~m}$ & Mirror size & $\phi 100 \times 60 \mathrm{~mm}$ \\
Recycling cavity length & $4.8 \mathrm{~m}$ & Beam-splitter size & $\phi 150 \times 40 \mathrm{~mm}$ \\
Asymmetry length & $0.85 \mathrm{~m}$ & Reflectivity of near mirror $^{a}$ & 0.988676 \\
Wavelength of light & $1.064 \mu \mathrm{m}$ & Reflectivity of end mirror $^{a}$ & 0.999875 \\
Sideband modulation & $15.235 \mathrm{MHz}$ & Refractive index $^{a}$ & 1.449750 \\
Modulation index & $0.35 \mathrm{rad}$ & Optical loss on coating & $30 \mathrm{ppm}$ \\
Injection laser power & $3 \mathrm{~W}$ & Pixel number $^{a}$ & $256 \times 256$ \\
Efficiency of photodetector & 0.8 & Calculation window size $^{b}$ & $200 \mathrm{~mm}$ \\
Beam-waist radius & $8.48 \mathrm{~mm}$ & & \\
\hline
\end{tabular}

${ }^{a}$ The calculation window size was chosen to reject aliasing.

${ }^{b}$ The design values of the reflectivity of the near mirror and the end mirror and the optical loss are $0.9880,0.9999$, and 40 ppm, respectively.

power, the reduction of the cavity reflectivity was mainly caused by light scattering owing to the imperfections of the high spatial frequency in the near mirrors and in the end mirrors. Although a slight decrease of the recycling gain was estimated if we considered the optical loss by pickoffs, the shot-noise sensitivity hardly changed.

We next examined whether a best rotation angle along the beam axis and combination of mirrors actually exists. Table 6 gives the numerical results for the case that one end mirror is rotated and that two of the end mirrors are exchanged. These results suggest that the rotation and combination of mirrors are not important for the polished TAMA mirrors.

From these results we conclude that these mirror performances will be able to achieve the target shotnoise sensitivity for the second phase of the TAMA.

\section{Conclusion}

In this paper we evaluated the performance of polished mirror surfaces for the TAMA by comparing the experimental results with a wave-front tracing simulation.

On the basis of an analysis of the phase maps of the TAMA mirrors, the curvature of the end mirrors and the recycling mirror have achieved their requirements. The FFT simulation showed that the quality of the polished TAMA mirrors is sufficient to achieve the requirements for the first phase of the TAMA. However, in that the performance of the optical coat-

Table 4. Results of the Experiment and the FFT Simulation

\begin{tabular}{lccc}
\hline \multicolumn{1}{c}{ Parameter } & Design $^{a}$ & Experiment & Simulation \\
\hline Cavity reflectivity & $95.7 \%^{b}$ & $96 \%$ & $91.3 \%$ \\
Finesse in in-line arm & 516 & 491 & 526 \\
Finesse in perpendicular arm & 516 & 512 & 528 \\
Contrast & $99.0 \%^{b}$ & $98.2 \%$ & $98.8 \%$ \\
Sensitivity $\mathrm{Hz}^{-1 / 2 c}$ & $2.0 \times 10^{-21}$ & $1.5 \times 10^{-20}$ & $4.9 \times 10^{-22}$ \\
\hline
\end{tabular}

${ }^{a}$ The design values were calculated by use of the design parameters.

${ }^{b}$ Estimation.

${ }^{c}$ The sensitivity is the strain sensitivity at $300 \mathrm{~Hz}$.

Table 5. Numerical Results of the FFT Simulation of the TAMA with a Recycling Mirror ${ }^{a}$

\begin{tabular}{|c|c|c|c|c|c|c|}
\hline \multirow[b]{3}{*}{ Parameter } & \multirow{2}{*}{\multicolumn{2}{|c|}{ Design }} & \multicolumn{4}{|c|}{ Simulation } \\
\hline & & & \multicolumn{2}{|c|}{ Without Phase Maps } & \multicolumn{2}{|c|}{ With Phase Maps } \\
\hline & Carrier & Sideband & Carrier & Sideband & Carrier & Sideband \\
\hline Cavity reflectivity & - & - & $\begin{array}{c}0.9468 \\
(0.9468)\end{array}$ & - & $\begin{array}{c}0.9069 \\
(0.9062)\end{array}$ & - \\
\hline Recycling gain & \multicolumn{2}{|c|}{$99.5 \%^{b}$} & 19 & 11 & 11 & 12 \\
\hline $\begin{array}{l}\text { Contrast } \\
\text { Shot-noise sensitivity } \\
\qquad \mathrm{Hz}^{-1 / 2 c}\end{array}$ & $1.6 \times 10^{-22}$ & $1.6 \times 10^{-22}$ & $1.1 \times 10^{-22}$ & $1.1 \times 10^{-22}$ & $1.6 \times 10^{-22}$ & $1.6 \times 10^{-22}$ \\
\hline
\end{tabular}

${ }^{a}$ The unparenthesized values are the total light power, and the parenthesized values are the $\mathrm{TEM}_{00}$ mode light. The reflectivity of the recycling mirror was optimized for the carrier beam in each calculation.

${ }^{b}$ Estimation.

${ }^{c}$ The shot-noise sensitivity is at a frequency of $300 \mathrm{~Hz}$. 
Table 6. Numerical Results When One End Mirror was Rotated along the Beam Axis and When Two of the End Mirrors were Exchanged ${ }^{a}$

\begin{tabular}{lccc}
\hline \multicolumn{1}{c}{ Parameter } & 90 Deg & 180 Deg & Exchange \\
\hline Recycling gain & 10 & 11 & 10 \\
Finesse in in-line arm & 524 & 526 & 528 \\
Finesse in perpendicular arm & 528 & 528 & 524 \\
Contrast (only carrier) & $99.8 \%$ & $99.8 \%$ & $99.7 \%$ \\
\hline
\end{tabular}

${ }^{a}$ The 90 Deg and 180 Deg columns show the cases in which the end mirror in the in-line arm was rotated by $90 \mathrm{deg}$ and $180 \mathrm{deg}$ along the beam axis. The Exchange column shows the case in which two of the end mirrors were exchanged.

ing is unknown, an evaluation of the coating performance is the next problem. In the second phase of the TAMA, which will implement a power-recycling mirror, the performance of the polished mirror surfaces will be able to achieve the target sensitivity for the TAMA. We concluded that the polished TAMA mirror surfaces have achieved their requirements. This evaluation will be useful for developing mirrors for the next generation of GW detectors, such as the Large-scale Cryogenic Gravitational wave Telescope $^{19}$ and the Advanced LIGO. ${ }^{20}$

\section{Appendix A: Newton's Ring Pattern}

Figure 4 shows an image of the Newton's ring pattern on the end mirror after subtraction of the global curvature. Because the intensity $(I)$ of the Newton's ring pattern is described as

$$
I(x, y)=\frac{I_{0}}{2}\left\{1+\cos \left[2 k \frac{\left(x^{2}+y^{2}\right)-a^{2}}{2 R}\right]\right\},
$$

we examined the pattern by fitting it to the function $I(x, y)=\alpha_{1}+\alpha_{2} \cos \left(\alpha_{3}+\alpha_{4} x+\alpha_{5} x^{2}+\alpha_{6} y+\alpha_{7} y^{2}\right)$,

where $I_{0}$ is the maximum intensity of the Newton's ring pattern, $k$ is the wave number of light, $a$ is the mirror radius, $R$ is the curvature radius of the mirror,

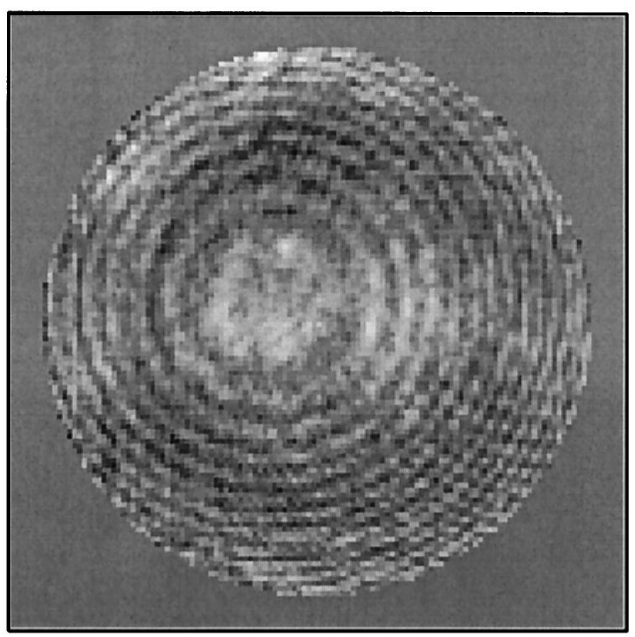

Fig. 4. Newton's ring pattern in an image of an end mirror. and $\alpha_{1}, \ldots, \alpha_{7}$ are fitting parameters. From this fitting, we recognized that the pattern was caused by a slight interference between the curved surface and the opposite face. This could not be removed by a refractive-matching oil during the measurement process.

\section{Appendix B: Calculation of Power Spectral Density}

The PSD for one-dimensional discrete data of surface imperfections is defined as ${ }^{15}$

$$
\begin{aligned}
P_{N}(m)= & \left\langle\frac{|\tilde{z}(m)|^{2}}{N \Delta x}\right\rangle, \\
\tilde{z}(m)= & \Delta x \sum_{n=0}^{N-1} z(n) \exp (-\mathrm{i} 2 \pi m n / N), \\
& -\frac{N}{2} \leq m \leq \frac{N}{2},
\end{aligned}
$$

where $P_{N}(m)$ is the PSD, $N$ is the number of discrete data of imperfections, $\Delta x$ is the sampling length, $z(n)$ is the amplitude at the $n$th position of the surface, and $\tilde{z}(m)$ is the $m$ th component of the Fourier transformation of $z$. Then the spatial frequency $\left(f_{m}\right)$ of the surface imperfections is defined as

$$
f_{m}=\frac{m}{N \Delta x}, \quad-\frac{N}{2} \leq m \leq \frac{N}{2} .
$$

In a practical calculation of the PSD, we need the periodogram approach. ${ }^{21}$ We first need a window function to avoid an impulse response. In this calculation, we used a Bartlett window,

$$
w(n)=1-\frac{2}{N-1}\left|n-\frac{N-1}{2}\right|, \quad 0 \leq n \leq N-1 .
$$

We next averaged the PSD to reduce the variance of the periodogram estimation. This is called the averaged PSD. In calculating the PSD for the mirror imperfections, several sets of one-dimensional surface data at different locations on the mirror surface were used. The PSD sets derived from each set of surface data were averaged. When we use $K$ sets of surface data, the averaged PSD $P_{\mathrm{av}}$ is given by

$$
\begin{aligned}
P_{\mathrm{av}}(m)= & \frac{1}{K} \sum_{j=1}^{K} P_{N}^{(j)}(m), \\
P_{N}^{(j)}(m)= & S \frac{\Delta x}{N}\left|\sum_{n=0}^{N-1} z^{(j)}(n) w(n) \exp (-\mathrm{i} 2 \pi m n / N)\right|^{2}, \\
& -\frac{N}{2} \leq m \leq \frac{N}{2},
\end{aligned}
$$


where $S$ shows the scaling factor determined by Parseval's theorem. That is, $S$ is determined from

$$
\frac{1}{2} P_{\mathrm{av}}(-N / 2)+\sum_{m=-N / 2+1}^{N / 2-1} P_{\mathrm{av}}(m)+\frac{1}{2} P_{\mathrm{av}}(N / 2)=\delta^{2},
$$

where $\delta^{2}$ shows the mean-square roughness of the mirror imperfections,

$$
\delta^{2}=\frac{1}{K N} \sum_{j=1}^{K} \sum_{n=0}^{N-1} z^{(j)}(n)^{2} .
$$

In the calculation in Fig. 2, we used an averaging method to overlap each set of data by one-half of their length by use of 30 data sets $(K=30)$. This averaging method yields a better PSD variance than the alternatives.

This study was carried out in an international collaboration between the TAMA and the LIGO projects. We express our appreciation to all the people involved. We also express our appreciation to P. Saha, one of the developers of the FFT simulator. We give particular thanks to P. Hello and J. Y. Vinet, the developers of the early FFT simulation code. We thank C. T. Taylor for useful advice in preparing this manuscript. This research was partially supported by a Grant-in-Aid for creative Basic Research from the Ministry of Education, Culture, Sports, Science, and Technology. It was also partially supported by a Grant-in-Aid for Creative Basic Research of the Ministry of Education (09NP0801) and the National Science Foundation under Cooperative Agreement PHY-9210038.

\section{References}

1. K. Tsubono, "300-m laser interferometer gravitational wave detector (TAMA300) in Japan," in Proceedings of the First Edoardo Amaldi Conference on Gravitational Wave Experiments (World Scientific, Singapore, 1995), pp. 112-114.

2. K. Kuroda, Y. Kozai, M. Fujimoto, M. Ohashi, R. Takahashi, T. Yamazaki, M. A. Barton, N. Kanda, Y. Saito, N. Kamikubota, Y. Ogawa, T. Suzuki, N. Kawashima, E. Mizuno, K. Tsubono, K. Kawabe, N. Mio, S. Moriwaki, A. Araya, K. Ueda, K. Nakagawa, T. Nakamura, and members of TAMA group, "Status of TAMA," in Proceedings of the International Conference on Gravitational Waves: Source and Detectors (World Scientific, Singapore, 1997), pp. 100-107.

3. M. Ando, K. Arai, R. Takahashi, G. Heinzel, S. Kawamura, D. Tatsumi, N. Kanda, H. Tagoshi, A. Araya, H. Asada, Y. Aso, M. A. Barton, M. Fujimoto, M. Fukushima, T. Futamase, K. Hayama, G. Horikoshi, H. Ishizuka, N. Kamikubota, K. Kawabe, N. Kawashima, Y. Kobayashi, Y. Kojima, K. Kondo, Y. Kozai, K. Kuroda, N. Matsuda, N. Mio, K. Miura, O. Miyakawa, S. M. Miyama, S. Miyoki, S. Moriwaki, M. Musha, S. Nagano, K. Nakagawa, T. Nakamura, K. Nakao, K. Numata, Y. Ogawa, M. Ohashi, N. Ohishi, S. Okutomi, K. Oohara, S. Otsuka, Y. Saito, M. Sasaki, S. Sato, A. Sekiya, M. Shibata, K. Somiya, T. Suzuki, A. Takamori, T. Tanaka, S. Taniguchi, S. Telada, K. Tochikubo, T. Tomaru, K. Tsubono, N. Tsuda, T. Uchiyama, A. Ueda, K. Ueda, K. Waseda, Y. Watanabe, H. Yakura, K. Yamamoto, and T. Yamazaki, "Stable operation of a 300-m laser interferometer with sufficient sensitivity to detect gravitational-wave events within our galaxy," Phys. Rev. Lett. 86, 3950-3954 (2001).

4. H. Tagoshi, N. Kanda, T. Tanaka, D. Tatsumi, S. Telada, M.
Ando, K. Arai, A. Araya, H. Asada, M. A. Barton, M. Fujimoto, M. Fukushima, T. Futamase, G. Heinzel, G. Horikoshi, H. Ishizuka, N. Kamikubota, K. Kawabe, S. Kawamura, N. Kawashima, Y. Kojima, Y. Kozai, K. Kuroda, N. Matsuda, S. Matsumura, S. Miki, N. Mio, O. Miyakawa, S. Miyama, S. Miyoki, E. Mizuno, S. Moriwaki, M. Musha, S. Nagano, K. Nakagawa, T. Nakamura, K. Nakao, K. Numata, Y. Ogawa, M. Ohashi, N. Ohishi, A. Okutomi, K. Oohara, S. Otsuka, Y. Saito, M. Sasaki, S. Sato, A. Sekiya, M. Shibata, K. Shirakata, K. Somiya, T. Suzuki, R. Takahashi, A. Takamori, S. Taniguchi, K. Tochikubo, T. Tomaru, K. Tsubono, N. Tsuda, T. Uchiyama, A. Ueda, K. Ueda, K. Ueda, K. Waseda, Y. Watanabe, H. Yakura, K. Yamamoto, and T. Yamazaki, "First search for gravitational waves from inspiraling compact binaries using TAMA300 data," Phys. Rev. D 63, 062001, 1-5 (2001).

5. A. Abramovici, W. E. Althouse, R. W. P. Drever, Y. Gursel, S. Kawamura, F. J. Raab, D. Shoemaker, L. Sievers, R. E. Spero, K. S. Thorne, R. E. Vogt, R. Weiss, S. E. Whitcomb, and M. E. Zucker, "LIGO: the Laser Interferometer GravitationalWave Observatory," Science 256, 325-333 (1992).

6. The VIRGO Collaboration, "VIRGO Final Design Report," VIRTRE-1000-13 (1997).

7. K. Danzmann, H. Lück, A. Rüdiger, R. Schilling, M. Schrempel, W. Winkler, J. Hough, G. P. Newton, N. A. Robertson, H. Ward, A. M. Campbell, J. E. Logan, D. I. Robertson, K. A. Strain, J. R. J. Bennett, V. Kose, M. Kühne, B. F. Schutz, D. Nicholson, J. Shuttleworth, H. Welling, P. Aufmuth, R. Rinkleff, A. Tünnermann, and B. Willke, "GEO 600: a 600-m laser interferometric gravitational wave antenna," in Proceedings of the First Edoardo Amaldi Conference on Gravitational Wave Experiments (World Scientific, Singapore, 1995), pp. $100-111$.

8. R. W. P. Drever, "Fabry-Perot cavity gravity-wave detectors," in The Detection of Gravitational Wave, D. G. Blair, ed. (Cambridge U. Press, Cambridge, UK, 1991), pp. 306-328.

9. P. Hello and J. Y. Vinet, "Numerical model of transient thermal effect in high power optical resonators," J. Phys. (Paris) I 3, 717-732 (1993).

10. B. Bochner, "Modeling the performance of interferometric gravitational-wave detectors with realistically imperfect optics," Ph.D. thesis (Massachusetts Institute of Technology, Cambridge, Mass., 1998).

11. P. Saha, "Fast estimation of transverse fields in high-finesse optical cavities," J. Opt. Soc. Am. A 14, 2195-2202 (1997).

12. N. Uehara, A. Ueda, K. Ueda, H. Sekiguchi, T. Mitake, K. Nakamura, N. Kitajima, and I. Kataoka, "Ultralow-loss mirror of the parts-in-10 $0^{6}$ level at $1064 \mathrm{~nm}$," Opt. Lett. 20, 530-532 (1995).

13. A. Ueda, K. Uehara, K. Uchisawa, K. Ueda, H. Sekiguchi, T. Mitake, K. Nakamura, N. Kitajima, and I. Kataoka, "Ultrahigh quality cavity with 1.5 ppm loss at $1064 \mathrm{~nm}$," Opt. Rev. 3, 369-372 (1996).

14. C. J. Walsh, A. J. Leistner, and B. F. Oreb, "Power spectral density analysis of optical substrates for gravitational-wave interferometry," Appl. Opt. 38, 4790-4801 (1999).

15. J. M. Elson and J. M. Benett, "Calculation of the power spectral density from surface profile data," Appl. Opt. 34, 201-208 (1995).

16. M. V. Berry, "Diffractals," J. Phys. A: Math. Gen. 12, 781797 (1979).

17. M. Born and E. Wolf, Principles of Optics, 5th ed. (Pergamon, New York, 1974).

18. S. Sato, S. Miyoki, M. Ohashi, M. Fujimoto, T. Yamazaki, M. Fukushima, A. Ueda, K. Ueda, K. Watanabe, K. Nakamura, K. Etoh, N. Kitajima, K. Ito, and I. Kataoka, "Loss factors of mirrors for a gravitational wave antenna," Appl. Opt. 38, 2880-2885 (1999).

19. K. Kuroda, M. Ohashi, S. Miyoki, D. Tatsumi, S. Sato, H. Ishizuka, M. Fujimoto, S. Kawamura, R. Takahashi, T. Yamazaki, K. Arai, M. Fukushima, K. Waseda, S. Telada, A. 
Ueda, T. Shintomi, A. Yamamoto, T. Suzuki, Y. Saito, T. Haruyama, N. Sato, K. Tsubono, K. Kawabe, M. Ando, K. Ueda, H. Yoneda, M. Musha, N. Mio, S. Moriwaki, A. Araya, N. Kanda, and M. E. Tobar, "Large-scale cryogenic gravitational wave telescope," Int. J. Mod. Phys. D 8, 557-579 (1999).

20. D. Shoemaker, "Advanced LIGO 2002-2006," presented at the
2001 Aspen Winter Conference on Gravitational Waves and Their Detection, Aspen, Colo., 4-10 February 2001, http:// www.ligo.caltech.edu/docs/G/G010020-00.pdf

21. W. H. Press, S. A. Teukolsky, W. T. Vetterling, and B. P. Flannery, "Numerical recipes in Fortran 77," (Cambridge U. Press, Cambridge, Mass., 1986). 